\title{
On the Initial Value Problem of Stochastic Evolution Equations in Hilbert Spaces
}

\author{
Xuping Zhang, Yongxiang Li, and Pengyu Chen \\ Department of Mathematics, Northwest Normal University, Lanzhou 730070, China \\ Correspondence should be addressed to Xuping Zhang; lanyu9986@126.com
}

Received 18 January 2016; Accepted 27 March 2016

Academic Editor: Jozef Banas

Copyright (C) 2016 Xuping Zhang et al. This is an open access article distributed under the Creative Commons Attribution License, which permits unrestricted use, distribution, and reproduction in any medium, provided the original work is properly cited.

\begin{abstract}
The present paper studies the initial value problem of stochastic evolution equations with compact semigroup in real separable Hilbert spaces. The existence of saturated mild solution and global mild solution is obtained under the situation that the nonlinear term satisfies some appropriate growth conditions. The results obtained in this paper improve and extend some related conclusions on this topic. An example is also given to illustrate that our results are valuable.
\end{abstract}

\section{Introduction}

In recent years, the stochastic differential equations have attracted great interest because of their practical applications in many areas such as physics, chemistry, economics, social sciences, finance, and other areas of science and engineering. For more details about stochastic differential equations we refer to the books by Sobczyk [1], Da Prato and Zabczyk [2], Grecksch and Tudor [3], Mao [4], and Liu [5]. One of the branches of stochastic differential equations is the theory of stochastic evolution equations. Since semilinear stochastic evolution equations are abstract formulations for many problems arising in the domain of engineering technology, biology, economic system, and so forth, stochastic evolution equations have attracted increasing attention in recent years and the existence, uniqueness, and asymptotic behavior of mild solutions to stochastic evolution equations have been considered by many authors; see [6-20] and the references therein. Taniguchi et al. [6] discussed the existence, uniqueness, pth moment, and almost sure Lyapunov exponents of mild solutions to a class of stochastic partial functional differential equations with finite delays by using semigroup methods. El-Borai et al. [7] studied exponentially asymptotic stability of stochastic differential equation in a real separable Hilbert space. More recently, Luo [8], Luo and Taniguchi [9], Bao et al. [10], and Sakthivel and Ren [11] discussed the exponential stability of mild solutions for stochastic partial differential equations by using the contraction mapping principle and stochastic integral technique, by the fixed point theorem, by introducing a suitable metric between the transition probability functions of mild solutions, and by using the stochastic analysis theory, respectively. Chang et al. [12-14] studied the existence and uniqueness of Stepanovlike almost automorphic mild solutions, the existence of square-mean almost automorphic mild solutions, and the existence and uniqueness of quadratic mean almost periodic mild solutions to nonlinear stochastic evolution equations in real separable Hilbert spaces, respectively. Moreover, the existence of mild solutions of stochastic evolution equations in Hilbert spaces has also been discussed in [15-20].

However, to the best of the authors' knowledge, most of the existing articles (see, e.g., [6, 12-19]) are only devoted to studying the local existence of mild solutions for stochastic evolution equations, and there are no results yet present on the existence of saturated mild solutions and global mild solutions for stochastic evolution equations in Hilbert spaces. Motivated by the abovementioned aspects, in this paper, by using Schauder's fixed point theorem, compact semigroup theory, and piecewise extension method, we investigate the existence of saturated mild solution and global mild solution for the initial value problem to a class of semilinear stochastic evolution equations in real separable Hilbert spaces. 


\section{Preliminaries}

Let $\mathbb{H}$ and $\mathbb{K}$ be two real separable Hilbert spaces and let $L(\mathbb{K}, \mathbb{H})$ be the space of all bounded linear operators from $\mathbb{K}$ into $\mathbb{H}$. For convenience, we will use the same notation $\|\cdot\|$ to denote the norms in $\mathbb{M}, \mathbb{K}$, and $L(\mathbb{K}, \mathbb{H})$ and use $(\cdot, \cdot)$ to denote the inner products of $\mathbb{M}$ and $\mathbb{K}$ without any confusion. Throughout this paper, we assume that $\left(\Omega, \mathscr{F}_{,}\left\{\mathscr{F}_{t}\right\}_{t \geq 0}, \mathbb{P}\right)$ is a complete filtered probability space satisfying the usual condition, which means that the filtration is a right continuous increasing family and $\mathscr{F}_{0}$ contains all $\mathbb{P}$-null sets of $\mathscr{F}$. Let $\left\{e_{k}, k \in \mathbb{N}\right\}$ be a complete orthonormal basis of $\mathbb{K}$. Suppose that $\{\mathbb{W}(t): t \geq 0\}$ is a cylindrical $\mathbb{K}$-valued Wiener process defined on the probability space $\left(\Omega, \mathscr{F},\left\{\mathscr{F}_{t}\right\}_{t \geq 0}, \mathbb{P}\right)$ with a finite trace nuclear covariance operator $Q \geq 0$, denote $\operatorname{Tr}(Q)=\sum_{k=1}^{\infty} \lambda_{k}=\lambda<\infty$, which satisfies that $Q e_{k}=\lambda_{k} e_{k}, k \in \mathbb{N}$. So, actually, $\mathbb{W}(t)=\sum_{k=1}^{\infty} \sqrt{\lambda_{k}} \mathbb{W}_{k}(t) e_{k}$, where $\left\{\mathbb{W}_{k}(t), k \in \mathbb{N}\right\}$ are mutually independent onedimensional standard Wiener processes. We further assume that $\mathscr{F}_{t}=\sigma\{W(s), 0 \leq s \leq t\}$ is the $\sigma$-algebra generated by W.

For $\varphi, \psi \in L(\mathbb{K}, \mathbb{H})$, we define $(\varphi, \psi)=\operatorname{Tr}\left(\varphi Q \psi^{*}\right)$, where $\psi^{*}$ is the adjoint of the operator $\psi$. Clearly, for any bounded operator $\psi \in L(\mathbb{K}, \mathbb{H})$,

$$
\|\psi\|_{Q}^{2}=\operatorname{Tr}\left(\psi Q \psi^{*}\right)=\sum_{k=1}^{\infty}\left\|\sqrt{\lambda_{k}} \psi e_{k}\right\| .
$$

If $\|\psi\|_{Q}^{2}<\infty$, then $\psi$ is called a $Q$-Hilbert-Schmidt operator.

In this paper, we consider the following initial value problem (IVP) of stochastic evolution equations:

$$
\begin{aligned}
d u(t)+A u(t) d t & =f(t, u(t)) d \mathbb{W}(t), \quad t \geq 0, \\
u(0) & =u_{0},
\end{aligned}
$$

where the state $u(\cdot)$ takes values in the real separable Hilbert space $\mathbb{H}, A: D(A) \subset \mathbb{H} \rightarrow \mathbb{H}$ is a closed linear operator, and $-A$ generates a compact $C_{0}$-semigroup $S(t)(t \geq 0)$ in $\mathbb{H}$; $f:[0, \infty) \times \mathbb{W} \rightarrow L(\mathbb{K}, \mathbb{W})$ is a continuous nonlinear mapping, $u_{0} \in \mathbb{H}$.

The collection of all strongly measurable, squareintegrable $\mathbb{H}$-valued random variables, denoted by $L^{2}(\Omega, \mathbb{H})$, is a Banach space equipped with the norm $\|u(\cdot)\|_{L^{2}}=(E \| u(\cdot$, $\left.\omega) \|^{2}\right)^{1 / 2}$, where the expectation $E$ is defined by $E u=\int_{\Omega} u(\omega) d \mathbb{P}$ with $\omega \in \Omega$. An important subspace of $L^{2}(\Omega$, $\mathbb{U})$ is given by

$$
L_{0}^{2}(\Omega, \mathbb{U})=\left\{u \in L^{2}(\Omega, \mathbb{U}) \mid u \text { is } \mathscr{F}_{0} \text {-measurable }\right\} .
$$

Let $J$ denote the closed subset of the interval $[0,+\infty)$. We denote by $C\left(J, L^{2}(\Omega, \mathbb{U})\right)$ the space of all continuous $\mathscr{F}_{t^{-}}$ adapted measurable processes from $J$ to $L^{2}(\Omega, \mathbb{H})$ satisfying $\sup _{t \in J} E\|u(t)\|^{2}<\infty$. Then it is easy to see that $C\left(J, L^{2}(\Omega, \mathbb{H})\right)$ is a Banach space endowed with the supnorm

$$
\|u\|_{C}=\left(\sup _{t \in J} E\|u(t)\|^{2}\right)^{1 / 2}
$$

Definition 1. By a mild solution of the IVP (2), we mean that a continuous $\mathscr{F}_{t}$-adapted stochastic process $u$ defined from $[0, \infty)$ to $L^{2}(\Omega, \mathbb{M})$ satisfies the following:

(i) $u_{0} \in L_{0}^{2}(\Omega, \mathbb{H})$,

(ii) $u(t) \in \mathbb{H}$ has cádlág paths on $t \in[0, \infty)$ almost surely and for each $t \in[0, \infty), u(t)$ satisfies the integral equation

$$
u(t)=S(t) u_{0}+\int_{0}^{t} S(t-s) f(s, u(s)) d \mathbb{W}(s) .
$$

\section{Main Results}

In this section, we prove the existence of saturated mild solution and global mild solution to the IVP (2). Firstly, in order to obtain the existence of saturated mild solution to the IVP (2), we impose the following assumption to the nonlinear term $f$.

$$
\begin{aligned}
& \left(H_{f}\right) \text { The function } f:[0,+\infty) \times \mathbb{M} \rightarrow L(\mathbb{K}, \mathbb{H}) \text { is } \\
& \text { continuous and the set }\left\{E\|f(t, u(t))\|^{2}: t \in J\right\} \text { is } \\
& \text { bounded for any bounded } E\|u(t)\|^{2} .
\end{aligned}
$$

Theorem 2. Assume that the condition $\left(H_{f}\right)$ is satisfied; then for every $u_{0} \in \mathbb{M}$ the IVP (2) has a saturated mild solution $u$ on a maximal interval of existence $[0, T)$. If $T<\infty$ then $\lim _{t \rightarrow T^{-}} E\|u(t)\|^{2}=\infty$.

Proof. We first prove the local existence of mild solution for the initial value problem (IVP) of stochastic evolution equations

$$
\begin{aligned}
d u(t)+A u(t) d t & =f(t, u(t)) d \mathbb{W}(t), \quad t \geq t_{0}, \\
u\left(t_{0}\right) & =x_{0}
\end{aligned}
$$

on interval $I:=\left[t_{0}, t_{0}+h\right]$, where $t_{0} \in[0, \infty), x_{0} \in \mathbb{H}$, and $h=$ $h\left(t_{0}, E\left\|x_{0}\right\|^{2}\right)>0$ will be given later. Consider the operator $F: C\left(I, L^{2}(\Omega, \mathbb{H})\right) \rightarrow C\left(I, L^{2}(\Omega, \mathbb{M})\right)$ defined by

$$
\begin{aligned}
(F u)(t)= & S\left(t-t_{0}\right) x_{0} \\
& +\int_{t_{0}}^{t} S(t-s) f(s, u(s)) d \mathbb{W}(s), \quad t \in I .
\end{aligned}
$$

From the continuity of nonlinear term $f$ one can easily see that the operator $F: C\left(I, L^{2}(\Omega, \mathbb{H})\right) \rightarrow C\left(I, L^{2}(\Omega, \mathbb{H})\right)$ is continuous. By Definition 1, the mild solution of the IVP (6) on $I$ is equivalent to the fixed point of operator $F$ defined by (7). Let

$$
\begin{aligned}
M\left(t_{0}\right) & =\sup \left\{\|S(t)\|: t_{0} \leq t \leq t_{0}+1\right\}, \\
R\left(t_{0}\right) & =2 M^{2}\left(t_{0}\right)\left(2 E\left\|x_{0}\right\|^{2}+1\right), \\
C\left(t_{0}\right) & =\sup \left\{E\|f(t, u(t))\|^{2}: E\|u(t)\|^{2} \leq R\left(t_{0}\right),\right. \\
t_{0} \leq & \left.t \leq t_{0}+1\right\} .
\end{aligned}
$$


Denote $\Theta=\left\{u \in C\left(I, L^{2}(\Omega, \mathbb{M})\right): E\|u(t)\|^{2} \leq R\left(t_{0}\right)\right\}$; then $\Theta$ is a closed ball in $C\left(I, L^{2}(\Omega, \mathbb{H})\right)$ with center $\theta$ and radius $R\left(t_{0}\right)$. Set

$$
h=h\left(t_{0}, E\left\|x_{0}\right\|^{2}\right):=\min \left\{1, \frac{E\left\|x_{0}\right\|^{2}+1}{\operatorname{Tr}(Q) C\left(t_{0}\right)}\right\} .
$$

For any $u \in \Theta$ and $t \in I$, by (7)-(9), we know that

$$
\begin{aligned}
E\|(F u)(t)\|^{2} \\
\leq 2 E\left\|S\left(t-t_{0}\right) x_{0}\right\|^{2} \\
\quad+2 E\left\|\int_{t_{0}}^{t} S(t-s) f(s, u(s)) d \mathbb{W}(s)\right\|^{2} \\
\leq 2 M^{2}\left(t_{0}\right) E\left\|x_{0}\right\|^{2} \\
\quad+2 \operatorname{Tr}(Q) M^{2}\left(t_{0}\right) \int_{t_{0}}^{t} E\|f(s, u(s))\|^{2} d s \\
\leq 2 M^{2}\left(t_{0}\right) E\left\|x_{0}\right\|^{2}+2 \operatorname{Tr}(Q) M^{2}\left(t_{0}\right) C\left(t_{0}\right) h \\
\leq R\left(t_{0}\right) .
\end{aligned}
$$

Therefore, $F u \in \Theta$. Thus, we proved that $F: \Theta \rightarrow \Theta$ is a continuous operator.

Now, we demonstrate that $F: \Theta \rightarrow \Theta$ is a compact operator. To prove this, we first show that $\{(F u)(t): u \in \Theta\}$ is relatively compact in $L^{2}(\Omega, \mathbb{H})$ for every $t \in I$. This is clear for $t=t_{0}$ since $\left\{(F u)\left(t_{0}\right): u \in \Theta\right\}=\left\{x_{0}\right\}$. For $t_{0}<t \leq t_{0}+h$, $0<\epsilon<t$, and $u \in \Theta$, we define the operator $F^{\epsilon}$ by

$$
\begin{aligned}
& \left(F^{\epsilon} u\right)(t) \\
& =S\left(t-t_{0}\right) x_{0}+\int_{t_{0}}^{t-\epsilon} S(t-s) f(s, u(s)) d \mathbb{W}(s) \\
& =S\left(t-t_{0}\right) x_{0} \\
& \quad+S(\epsilon) \int_{t_{0}}^{t-\epsilon} S(t-s-\epsilon) f(s, u(s)) d \mathbb{W}(s)
\end{aligned}
$$

Since $S(t)$ is compact for every $t>0$, the set $\left\{\left(F^{\epsilon} u\right)(t)\right.$ : $u \in \Theta\}$ is relatively compact in $L^{2}(\Omega, \mathbb{H})$ for every $\epsilon \in(0, t)$. Moreover, for every $u \in \Theta$, by (7), (8), and (11) we get that

$$
\begin{aligned}
& E\left\|(F u)(t)-\left(F^{\epsilon} u\right)(t)\right\|^{2} \\
& \quad \leq E\left\|\int_{t-\epsilon}^{t} S(t-s) f(s, u(s)) d \mathbb{W}(s)\right\|^{2} \\
& \quad \leq \operatorname{Tr}(Q) M^{2}\left(t_{0}\right) \int_{t-\epsilon}^{t} E\|f(s, u(s))\|^{2} d s \\
& \quad \leq \operatorname{Tr}(Q) M^{2}\left(t_{0}\right) C\left(t_{0}\right) \epsilon .
\end{aligned}
$$

Therefore, we have proved that there are relatively compact sets arbitrarily close to the set $\{(F u)(t): u \in \Theta\}$ in $L^{2}(\Omega, \mathbb{H})$ for $t_{0}<t \leq t_{0}+h$. Hence, the set $\{(F u)(t): u \in \Theta\}$ is also relatively compact in $L^{2}(\Omega, \mathbb{U})$ for $t_{0}<t \leq t_{0}+h$. And therefore, we have the compactness of $\{(F u)(t): u \in \Theta\}$ in $L^{2}(\Omega, \mathbb{M})$ for all $t \in I$. We continue to show that $F(\Theta)$ is an equicontinuous family of functions in $C\left(I, L^{2}(\Omega, \mathbb{H})\right)$. For any $u \in \Theta$ and $t_{0} \leq t_{1}<t_{2} \leq t_{0}+h$, we have

$$
\begin{aligned}
& E\left\|(F u)\left(t_{2}\right)-(F u)\left(t_{1}\right)\right\|^{2} \leq 3 E \| S\left(t_{2}-t_{0}\right) x_{0}-S\left(t_{1}\right. \\
& \left.-t_{0}\right) x_{0}\left\|^{2}+3 E\right\| \int_{t_{1}}^{t_{2}} S\left(t_{2}-s\right) f(s, u(s)) d \mathbb{W}(s) \|^{2} \\
& +3 E \| \int_{t_{0}}^{t_{1}}\left(S\left(t_{2}-s\right)-S\left(t_{1}-s\right)\right) \\
& \cdot f(s, u(s)) d \mathbb{W}(s)\left\|^{2} \leq 3 E\right\|\left(S\left(t_{2}-t_{0}\right)\right. \\
& \left.-S\left(t_{1}-t_{0}\right)\right) x_{0} \|^{2}+3 \operatorname{Tr}(Q) M^{2}\left(t_{0}\right) \\
& \cdot \int_{t_{1}}^{t_{2}} E\|f(s, u(s))\|^{2} d s+3 \operatorname{Tr}(Q) \int_{t_{0}}^{t_{1}} \| S\left(t_{2}-s\right) \\
& \quad-S\left(t_{1}-s\right)\left\|^{2} E\right\| f(s, u(s)) \|^{2} d s \\
& \quad \leq 3 E\left\|\left(S\left(t_{2}-t_{0}\right)-S\left(t_{1}-t_{0}\right)\right) x_{0}\right\|^{2}+3 \operatorname{Tr}(Q) \\
& \quad M^{2}\left(t_{0}\right) C\left(t_{0}\right)\left(t_{2}-t_{1}\right)+3 \operatorname{Tr}(Q) C\left(t_{0}\right) \\
& \cdot \int_{t_{0}}^{t_{1}}\left\|S\left(t_{2}-s\right)-S\left(t_{1}-s\right)\right\|^{2} d s .
\end{aligned}
$$

Since $S\left(t-t_{0}\right) x_{0}$ is continuous on $\left[t_{0}, t_{0}+h\right]$, it is uniformly continuous, and therefore the first term of the right-hand side of (13) tends to zero as $t_{2}-t_{1} \rightarrow 0$. It is obvious that the second term of the right-hand side of (13) tends to zero as $t_{2}-t_{1} \rightarrow 0$. Notice that $S(t)$ is compact for $t>0$; we know that $S(t)$ is continuous by operator norm for $t>0$. Combining this fact with the Lebesgue dominated convergence theorem we know that the third term of the right-hand side of (13) tends to zero as $t_{2}-t_{1} \rightarrow 0$. Therefore, $E\left\|(F u)\left(t_{2}\right)-(F u)\left(t_{1}\right)\right\|^{2}$ tends to zero independently of $u \in \Theta$ as $t_{2}-t_{1} \rightarrow 0$, which means that $F: \Theta \rightarrow \Theta$ is equicontinuous. Hence by the ArzelaAscoli theorem one has that $F: \Theta \rightarrow \Theta$ is a compact operator. Therefore, by Schauder fixed point theorem we obtain that $F$ has at least one fixed point $u\left(t_{0}\right) \in \Theta$, which is in turn a mild solution of the IVP (6) on the interval $\left[t_{0}, t_{0}+h\right]$.

From the local existence of mild solutions we have just proved it follows that there exists interval $\left[0, h_{0}\right]$ such that the IVP (2) has a mild solution $u \in C\left(\left[0, h_{0}\right], L^{2}(\Omega, \mathbb{H})\right)$, and it can be extended to a large interval $\left[0, h_{0}+h\right]$ with $h>0$ by defining $u(t)=v(t)$ on $\left[h_{0}, h_{0}+h\right]$, where $v(t)$ is the mild solution of the initial value problem

$$
\begin{aligned}
& d v(t)+A v(t) d t=f(t, v(t)) d \mathbb{W}(t), \\
& h_{0} \leq t \leq h_{0}+h \text {, } \\
& v(0)=u\left(h_{0}\right),
\end{aligned}
$$

where $h$ depends only on $E\left\|u\left(h_{0}\right)\right\|^{2}, R\left(h_{0}\right)$, and $C\left(h_{0}\right)$. Therefore, repeating the above procedure and using 
the methods of steps, we can prove that there exists a maximal interval $[0, T)$ such that $u \in C\left([0, T), L^{2}(\Omega, \mathbb{H})\right)$ is a saturated mild solution of the IVP (2).

Next, we show that if $T<\infty$ then $\lim _{t \rightarrow T^{-}} E\|u(t)\|^{2}=$ $\infty$. To do so we first prove that $T<\infty$ implies $\lim \sup _{t \rightarrow T^{-}} E\|u(t)\|^{2}=\infty$. Indeed, if $T<\infty$ and $\lim \sup _{t \rightarrow T^{-}} E\|u(t)\|^{2}<\infty$, then there exists a constant $0<$ $R(T)<\infty$ such that $\sup _{0 \leq t<T} E\|u(t)\|^{2} \leq R(T)$. Denote $M(T)=\sup \{\|S(t)\|: 0 \leq t \leq T+1\}$ and $C(T)=\sup \{E \| f(t$, $\left.u(t))\left\|^{2}: E\right\| u(t) \|^{2} \leq R(T), 0 \leq t \leq T+1\right\}$. For $0<t^{\prime}<t^{\prime \prime}<T$, we have

$$
\begin{aligned}
& E\left\|u\left(t^{\prime \prime}\right)-u\left(t^{\prime}\right)\right\|^{2} \leq 3 E\left\|S\left(t^{\prime \prime}\right) x_{0}-S\left(t^{\prime}\right) x_{0}\right\|^{2} \\
& +3 E\left\|\int_{t^{\prime}}^{t^{\prime \prime}} S\left(t^{\prime \prime}-s\right) f(s, u(s)) d \mathbb{W}(s)\right\|^{2} \\
& +3 E \| \int_{0}^{t^{\prime}}\left(S\left(t^{\prime \prime}-s\right)-S\left(t^{\prime}-s\right)\right) \\
& \cdot f(s, u(s)) d \mathbb{W}(s)\left\|^{2} \leq\right\| S\left(t^{\prime \prime}\right)-S\left(t^{\prime}\right) \|^{2} \\
& +3 E\left\|x_{0}\right\|^{2}+3 \operatorname{Tr}(Q) M^{2}(T) C(T)\left(t^{\prime \prime}-t^{\prime}\right) \\
& +3 \operatorname{Tr}(Q) C(T) \int_{0}^{t^{\prime}}\left\|S\left(t^{\prime \prime}-s\right)-S\left(t^{\prime}-s\right)\right\|^{2} d s .
\end{aligned}
$$

The right-hand side of (15) tends to zero as $t^{\prime}, t^{\prime \prime} \rightarrow T^{-}$as a consequence of the continuity of $S(t)$ in the uniform operator topology for $t>0$ which in turn follows the compactness of $S(t)$ for $t>0$. Therefore, $\lim _{t \rightarrow T^{-}} u(t)=u(T)$ exists. Let $\lim _{t \rightarrow T^{-}} u(t)=x_{1}$; by the local existence of mild solutions we proved above we know that the stochastic evolution equation

$$
\begin{aligned}
d u(t)+A u(t) d t & =f(t, u(t)) d \mathbb{W}(t), \quad t \geq T, \\
u(T) & =x_{1}
\end{aligned}
$$

presents a mild solution on $[T, T+h]$, where $h>0$ is a constant. This means that the mild solution $u$ of the IVP (2) can be extended beyond $T$, which contradicts with $u \in$ $C\left([0, T), L^{2}(\Omega, \mathbb{H})\right)$ being a saturated mild solution of the IVP (2). Therefore, the assumption $T<\infty$ implies that $\lim \sup _{t \rightarrow T^{-}} E\|u(t)\|^{2}=\infty$. To conclude the proof we will show that $T<\infty$ implies $\lim _{t \rightarrow T^{-}} E\|u(t)\|^{2}=\infty$. If this is not true, then there exist a constant $R>0$ and a sequence $t_{n} \uparrow T$ such that $E\left\|u\left(t_{n}\right)\right\|^{2} \leq R$ for all $n$. Let $M=\sup \{\|S(t)\|$ : $0 \leq t \leq T\}$ and $C=\sup \left\{E\|f(t, u(t))\|^{2}: E\|u(t)\|^{2} \leq\right.$ $\left.2 M^{2}(R+1), 0 \leq t \leq T\right\}$. By the fact that $t \rightarrow E\|u(t)\|^{2}$ is continuous and $\lim \sup _{t \rightarrow T^{-}} E\|u(t)\|^{2}=\infty$ one can find a sequence $\left\{h_{n}\right\}\left(0<h_{n}<T-t_{n}\right)$ such that $E\|u(t)\|^{2} \leq$ $2 M^{2}(R+1)$ for $t_{n} \leq t \leq t_{n}+h_{n}$ and $E\left\|u\left(t_{n}+h_{n}\right)\right\|^{2}=2 M^{2}(R+1)$. By direct calculation, we have

$$
\begin{aligned}
& 2 M^{2}(R+1)=E\left\|u\left(t_{n}+h_{n}\right)\right\|^{2} \\
& \quad \leq 2 E\left\|S\left(h_{n}\right) u\left(t_{n}\right)\right\|^{2}
\end{aligned}
$$

$$
\begin{aligned}
& \quad+2 E\left\|\int_{t_{n}}^{t_{n}+h_{n}} S\left(t_{n}+h_{n}-s\right) f(s, u(s)) d \mathbb{W}(s)\right\|^{2} \\
& \leq 2 M^{2} R+2 \operatorname{Tr}(Q) M^{2} C h_{n} \longrightarrow 2 M^{2} R
\end{aligned}
$$$$
\text { as } n \longrightarrow \infty \text {. }
$$

From (17) we conclude that $M^{2} \leq 0$, which contradicts with the definition of $M$. Therefore, we have proved that if $T<$ $\infty$ then $\lim _{t \rightarrow T^{-}} E\|u(t)\|^{2}=\infty$. This completes the proof of Theorem 2.

Next, we discuss the existence of global mild solution to the IVP (2). To this end, we need to replace the assumption $\left(H_{f}\right)$ by the following assumption:
$\left(H_{f}\right)^{\prime}$ The function $f:[0,+\infty) \times \mathbb{W} \rightarrow L(\mathbb{K}, \mathbb{M})$ is continuous and there exist two functions $K_{1}, K_{2} \in$ $C([0, \infty),[0, \infty))$ such that

$$
\begin{aligned}
& E\|f(t, u(t))\|^{2} \leq K_{1}(t) E\|u(t)\|^{2}+K_{2}(t) \\
& \qquad \text { for } 0 \leq t<\infty, u \in \mathbb{H} .
\end{aligned}
$$

Theorem 3. Assume that the condition $\left(H_{f}\right)^{\prime}$ is satisfied; then for every $u_{0} \in \mathbb{H}$ the IVP (2) has a global mild solution $u \in$ $C\left([0, \infty), L^{2}(\Omega, \mathbb{H})\right)$.

Proof. It is obvious that $\left(H_{f}\right)^{\prime} \Rightarrow\left(H_{f}\right)$. Therefore, by Theorem 2 we know that the IVP (2) has a saturated mild solution $u$ on a maximal interval of existence $[0, T)$. If $T=\infty$, then the result has been proved. If $T<\infty$, by the proof process of Theorem 2 one can see that the IVP (2) has a global mild solution if $E\|u(t)\|^{2}<+\infty$ for every $t$ in the interval of existence of $u$. Therefore, in order to prove Theorem 3 we only need to prove $E\|u(t)\|^{2}<+\infty$ for every $t \in[0, T)(T<\infty)$. Let

$$
\begin{aligned}
& \bar{M}=\sup _{t \in[0, T]}\|S(t)\|, \\
& C_{1}=\max _{t \in[0, T]} C_{1}(t), \\
& C_{2}=\max _{t \in[0, T]} C_{2}(t) .
\end{aligned}
$$

By Definition 1, the assumption $\left(H_{f}\right)^{\prime}$, and (19) we know that for any $t \in[0, T)$,

$$
\begin{aligned}
E\|u(t)\|^{2} \leq & 2 E\left\|S(t) u_{0}\right\|^{2} \\
& +2 E\left\|\int_{0}^{t} S(t-s) f(s, u(s)) d \mathbb{W}(s)\right\|^{2} \\
\leq & 2 \bar{M} E\left\|u_{0}\right\|^{2} \\
& +2 \operatorname{Tr}(Q) \bar{M}^{2} \int_{0}^{t} E\|f(s, u(s))\|^{2} d s
\end{aligned}
$$




$$
\begin{aligned}
\leq & 2 \bar{M} E\left\|u_{0}\right\|^{2}+2 \operatorname{Tr}(Q) \bar{M}^{2} K_{2} T \\
& +2 \operatorname{Tr}(Q) \bar{M}^{2} K_{1} \int_{0}^{t} E\|u(s)\|^{2} d s \\
:= & N_{1}+N_{2} \int_{0}^{t} E\|u(s)\|^{2} d s,
\end{aligned}
$$

where

$$
\begin{aligned}
& N_{1}=2 \bar{M} E\left\|u_{0}\right\|^{2}+2 \operatorname{Tr}(Q) \bar{M}^{2} K_{2} T, \\
& N_{2}=2 \operatorname{Tr}(Q) \bar{M}^{2} K_{1} .
\end{aligned}
$$

Therefore, by (20) and Gronwall's inequality, we know that

$$
E\|u(t)\|^{2} \leq N_{1} e^{N_{2} T}<+\infty, \text { for } t \in[0, T) .
$$

This completes the proof of Theorem 3.

\section{An Application}

In this section, we give an example to illustrate the applicability of our main results. Let $U \subset \mathbb{R}^{N}$ be a bounded domain with a sufficiently smooth boundary $\partial U$. We consider the initial-boundary value problem of stochastic parabolic partial differential equation

$$
\begin{aligned}
d w(x, t)-\Delta w(x, t) d t & =\frac{\sin (\pi t)}{\sqrt{2}(1+|w(x, t)|)} d \mathbb{W}(t), \\
x \in U, t \geq 0, & \\
\left.w\right|_{\partial U} & =0, \\
w(x, 0) & =\varphi(x), \quad x \in U,
\end{aligned}
$$

where $\Delta$ is the Laplace operator, $\mathbb{W}(t)$ denotes a onedimensional standard cylindrical Wiener process defined on a stochastic space $\left(\Omega, \mathscr{F},\left\{\mathscr{F}_{t}\right\}_{t \geq 0}, \mathbb{P}\right)$, and $\varphi \in L^{2}(U)$ is $\mathscr{F}_{0^{-}}$ measurable.

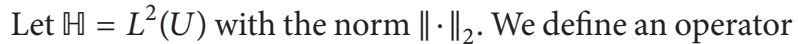
$A$ in Hilbert space $\mathbb{H}$ by

$$
\begin{aligned}
D(A) & =H^{2}(U) \cap H_{0}^{1}(U), \\
A w & =-\Delta w .
\end{aligned}
$$

It is well known from $[21,22]$ that $-A$ generates a compact $C_{0}$-semigroup $S(t)(t \geq 0)$ in $\mathbb{H}$. Let $u(t)=w(\cdot, t)$, $f(t, u(t))=\sin (\pi t) / \sqrt{2}(1+|w(\cdot, t)|)$, and $u_{0}=\varphi(\cdot)$; then the initial-boundary value problem of stochastic parabolic partial differential equation (23) can be transformed into the abstract form of IVP (2).

Theorem 4. The initial-boundary value problem of stochastic parabolic partial differential equation (23) has a global mild solution $w \in C(\bar{U} \times[0, \infty))$.

Proof. From the definition of nonlinear term $f$, we can easily verify that the assumption $\left(H_{f}\right)^{\prime}$ is satisfied with $K_{1}(t)=$ $K_{2}(t)=1$. Therefore, our conclusion follows from Theorem 3 . This completes the proof.

\section{Competing Interests}

The authors declare that they have no competing interests.

\section{Acknowledgments}

Research supported by The Science Research Project for Colleges and Universities of Gansu Province (2015A-213, 2015A-003), NNSF of China (11501455, 11261053), and Project of NWNU-LKQN-14-3.

\section{References}

[1] K. Sobczyk, Stochastic Differential Equations with Applications to Physics and Engineering, Kluwer Academic, London, UK, 1991.

[2] G. Da Prato and J. Zabczyk, Stochastic Equations in Infinite Dimensions, Cambridge University Press, Cambridge, UK, 1992.

[3] W. Grecksch and C. Tudor, Stochastic Evolution Equations: A Hilbert Space Approach, Akademie Verlag, Berlin, Germany, 1995.

[4] X. Mao, Stochastic Differential Equations and Their Applications, Horwood, Chichester, UK, 1997.

[5] K. Liu, Stability of Infinite Dimensional Stochastic Differential Equations with Applications, Chapman \& Hall, London, UK, 2006.

[6] T. Taniguchi, K. Liu, and A. Truman, "Existence, uniqueness, and asymptotic behavior of mild solutions to stochastic functional differential equations in Hilbert spaces," Journal of Differential Equations, vol. 181, no. 1, pp. 72-91, 2002.

[7] M. M. El-Borai, O. L. Moustafa, and H. M. Ahmed, "Asymptotic stability of some stochastic evolution equations," Applied Mathematics and Computation, vol. 144, no. 2-3, pp. 273-286, 2003.

[8] J. Luo, "Fixed points and exponential stability of mild solutions of stochastic partial differential equations with delays," Journal of Mathematical Analysis and Applications, vol. 342, no. 2, pp. 753-760, 2008.

[9] J. Luo and T. Taniguchi, "Fixed points and stability of stochastic neutral partial differential equations with infinite delays," Stochastic Analysis and Applications, vol. 27, no. 6, pp. 1163-1173, 2009.

[10] J. Bao, Z. Hou, and C. Yuan, "Stability in distribution of mild solutions to stochastic partial differential equations," Proceedings of the American Mathematical Society, vol. 138, no. 6, pp. 2169-2180, 2010.

[11] R. Sakthivel and Y. Ren, "Exponential stability of second-order stochastic evolution equations with Poisson jumps," Communications in Nonlinear Science and Numerical Simulation, vol. 17, no. 12, pp. 4517-4523, 2012.

[12] Y.-K. Chang, Z.-H. Zhao, G. M. N’Guérékata, and R. Ma, "Stepanov-like almost automorphy for stochastic processes and applications to stochastic differential equations," Nonlinear Analysis. Real World Applications, vol. 12, no. 2, pp. 1130-1139, 2011.

[13] Y.-K. Chang, Z.-H. Zhao, and G. N’Guérékata, “A new composition theorem for square-mean almost automorphic functions and applications to stochastic differential equations," Nonlinear Analysis. Theory, Methods \& Applications, vol. 74, no. 6, pp. 2210-2219, 2011. 
[14] Y.-K. Chang, R. Ma, and Z.-H. Zhao, "Almost periodic solutions to a stochastic differential equation in Hilbert spaces," Results in Mathematics, vol. 63, no. 1-2, pp. 435-449, 2013.

[15] J. Bao and Z. Hou, "Existence of mild solutions to stochastic neutral partial functional differential equations with nonLipschitz coefficients," Computers \& Mathematics with Applications, vol. 59, no. 1, pp. 207-214, 2010.

[16] Y. Ren, Q. Zhou, and L. Chen, "Existence, uniqueness and stability of mild solutions for time-dependent stochastic evolution equations with Poisson jumps and infinite delay," Journal of Optimization Theory and Applications, vol. 149, no. 2, pp. 315331, 2011.

[17] Z. Yan and X. Yan, "Existence of solutions for impulsive partial stochastic neutral integrodifferential equations with statedependent delay," Collectanea Mathematica, vol. 64, no. 2, pp. 235-250, 2013.

[18] J. Cui, L. Yan, and X. Wu, "Nonlocal Cauchy problem for some stochastic integro-differential equations in Hilbert spaces," Journal of the Korean Statistical Society, vol. 41, no. 3, pp. 279290, 2012.

[19] P. Chen, Y. Li, and X. Zhang, "On the initial value problem of fractional stochastic evolution equations in Hilbert spaces," Communications on Pure and Applied Analysis, vol. 14, no. 5, pp. 1817-1840, 2015.

[20] P. Chen and Y. Li, "Nonlocal Cauchy problem for fractional stochastic evolution equations in Hilbert spaces," Collectanea Mathematica, vol. 66, no. 1, pp. 63-76, 2015.

[21] A. Pazy, Semigroups of Linear Operators and Applications to Partial Differential Equations, Springer, Berlin, Germany, 1983.

[22] I. I. Vrabie, $\mathrm{C}_{0}$-Semigroups and Applications, vol. 191 of NorthHolland Mathematics Studies, Elsevier, Amsterdam, Netherlands, 2003. 


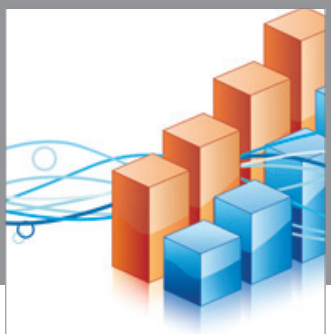

Advances in

Operations Research

vatem alat4

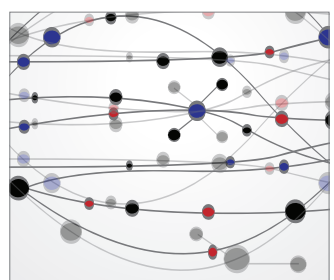

\section{The Scientific} World Journal
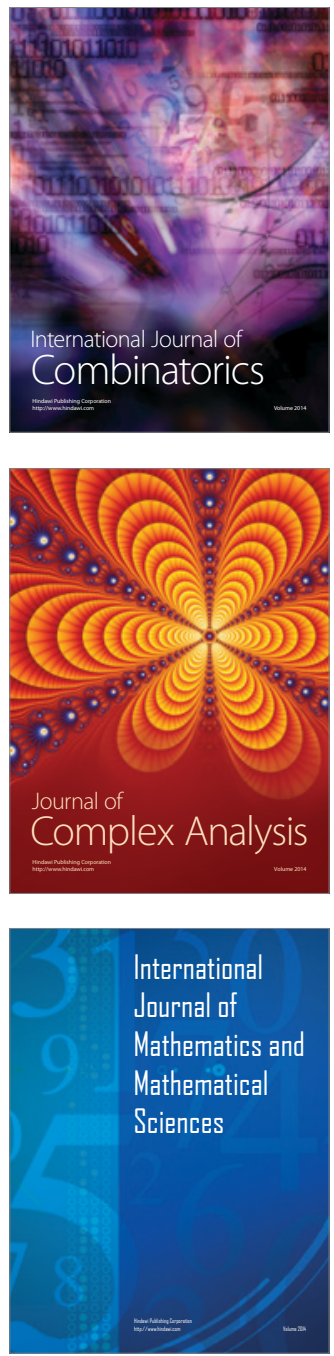
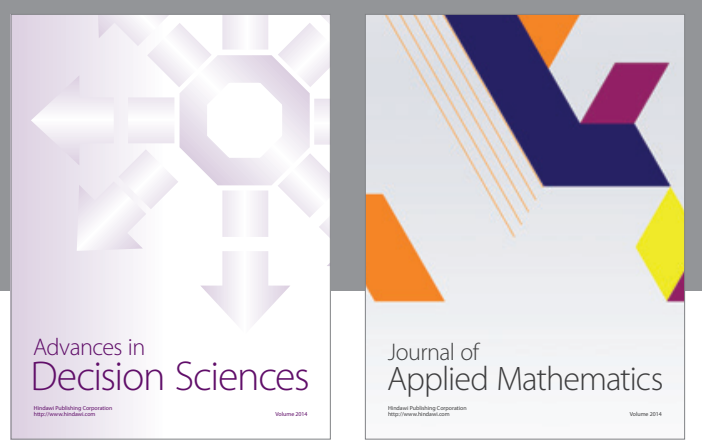

Algebra

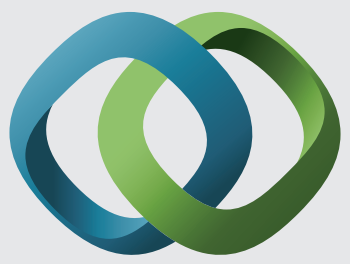

\section{Hindawi}

Submit your manuscripts at

http://www.hindawi.com
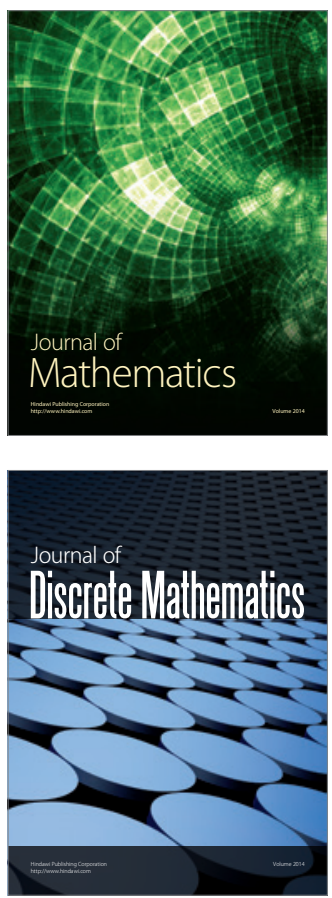

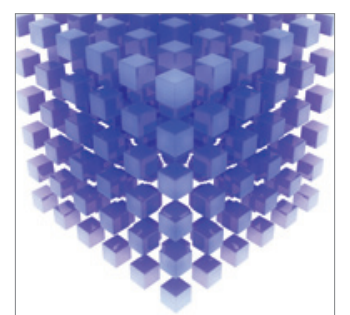

Mathematical Problems in Engineering
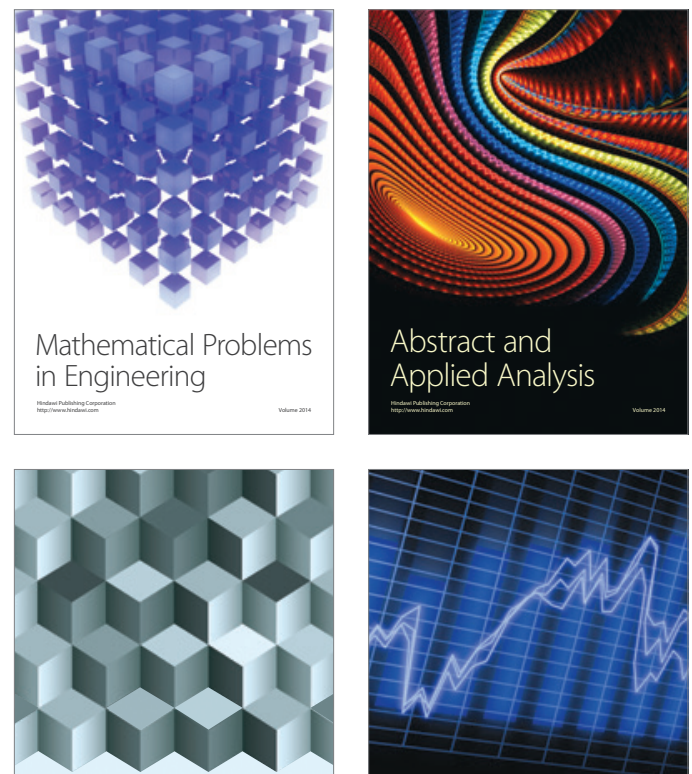

Journal of

Function Spaces

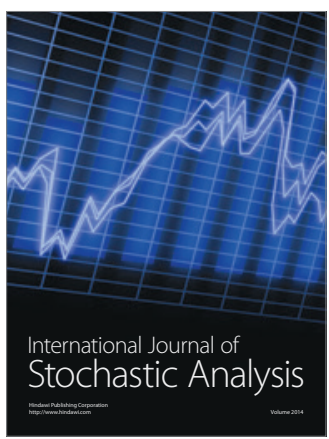

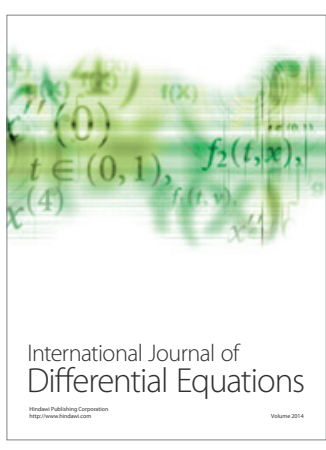
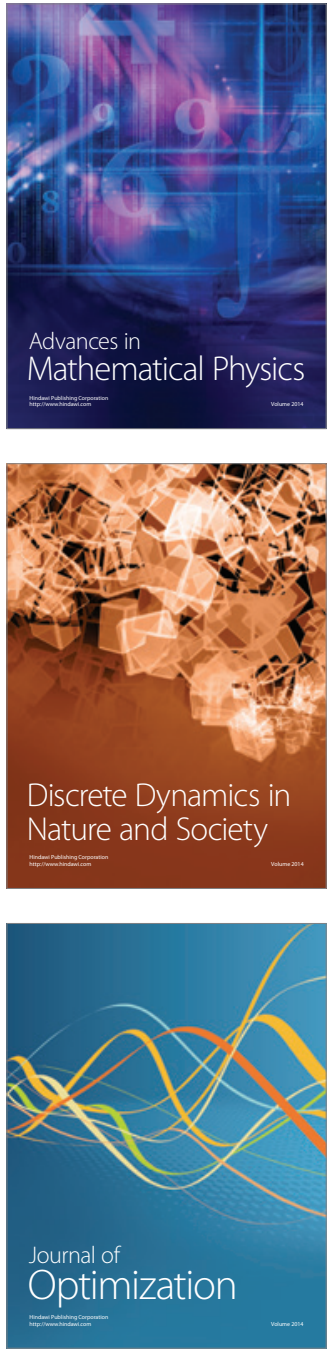